\title{
Vulnerabilidad estructural ante riesgo sísmico de las viviendas de la subcuenca Chucchun - Carhuaz
}

Structural vulnerability to the seismic risk of homes in the Chucchun sub-basin Carhuaz

\section{Rosa Deifilia Rodríguez Anaya ${ }^{1}$}

\section{RESUMEN}

La investigación tuvo como objetivos identificar las características del territorio y de los indicadores que influyen en la vulnerabilidad estructural de las viviendas ante el riesgo símico. En la metodología se aplico el diseño no experimental transeccional, usando como instrumento un cuestionario para la aplicación de encuestas a la muestra de 343 viviendas; para determinar el nivel de vulnerabilidad de las viviendas se utilizó el Proceso de Análisis Jerárquico (PAJ). Los hallazgos evidencian que el territorio de la subcuenca Chucchun, por las características de la litología, pendiente y susceptibilidad o exposición presentan condiciones de vulnerabilidad alta y muy alta; los resultados de las encuestas evidencian que las viviendas presentan condiciones de vulnerabilidad y ante la ocurrencia del riesgo sísmico, las viviendas son altamente vulnerables.

Palabras clave: vulnerabilidad estructural; viviendas; riesgo sísmico; subcuenca.

\footnotetext{
ABSTRACT

The research had as objectives to identify the characteristics of the territory and the indicators that influence the structural vulnerability of the houses to the simian risk . In the methodology, the non-experimental transectional design was applied, using as a

1 Universidad Nacional Santiago Antúnez de Mayolo. Huaraz, Perú.

(C) Los autores. Este artículo es publicado por la Revista Aporte Santiaguino de la Universidad Nacional Santiago Antúnez de Mayolo. Este es un artículo de acceso abierto, distribuido bajo los términos de la Licencia Creative Commons Atribución-NoComercial-CompartirIgual 4.0 Internacional. (http://creativecommons.org/licenses/ by-nc-sa/4.0/), que permite el uso no comercial, distribución y reproducción en cualquier medio, siempre que la obra original sea debidamente citada.
} 
tool a questionnaire for the application of surveys to the sample of 343 dwellings; The Analysis Hierarchical Process (AHP) was used to determine the level of vulnerability of the houses. The findings show that the territory of the Chucchun sub-basin, due to the characteristics of the lithology, slope and susceptibility or exposure, presents conditions of high and very high vulnerability; the results of the surveys show that the houses present conditions of vulnerability and before the occurrence of the seismic risk, the houses are highly vulnerable.

Keywords: Structural vulnerability; households; seismic risk; sub-basin

\section{INTRODUCCIÓN}

Las evidencias históricas, muestran que el territorio peruano ha sufrido grandes catástrofes a lo largo de su historia, como producto de eventos de gran magnitud e intensidad, como los terremotos, el fenómeno El Niño, inundaciones, olas de frío intenso, sequías, etc. que en muchas ocasiones causaron la desaparición de pueblos y obligó a la migración de los sobrevivientes. Un ejemplo reciente fue el sismo y posterior aluvión que sepultó a la ciudad de Yungay en el año 1970.

Según el Instituto Geofísico del Perú (2011) el mayor número y los más grandes sismos se han producido en el llamado Cinturón de Fuego del Océano Pacífico que considera a los países que bordean dicho océano; ejemplos son los sismos ocurridos en la última década en América del Sur: Arequipa en 2001 con 8,2 grados de magnitud; Pisco en 2007, con 8,0 grados de magnitud; y Chile en 2010, con una magnitud de 8,8 grados (ver figura 1 ).

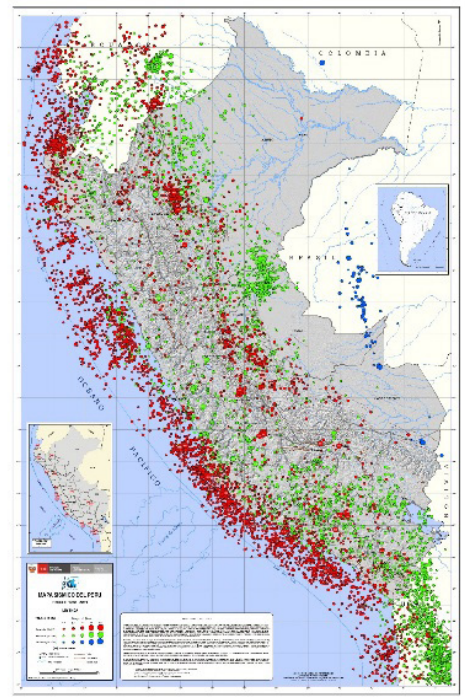

Figura 1. Mapa sísmico del Perú- IGP 2011 
De otro lado, la dimensión del problema de vulnerabilidad del Perú ante desastres es significativa, el Perú está identificado como uno de los países con mayor vulnerabilidad y a sufrir desastres debido a su ubicación dentro del Cinturón de Fuego del Pacífico, donde ocurre el 85\% de todos los sismos del mundo, según, el IGP, nuestro país cuenta con redes sísmicas y de GPS, cuya información permite identificar la ubicación de las asperezas (áreas de ruptura) que darían origen en el futuro, a los próximos grandes sismos frente a múltiples amenazas naturales en todo el continente, por lo tanto se debe mejorar el desempeño en componentes claves de la gestión de riesgos: gobernabilidad, protección financiera, identificación de riesgo, reducción de riesgo, y manejo de desastre, partiendo del fortalecimiento de las instituciones, comunidades y organizaciones que fundamentalmente sufren los impactos de estos desastres y que las inversiones que se hagan en la gestión del riesgo se evalúen en función de eficiencia y de acuerdo a la política presupuestaria del estado, medida por productos que puedan ser evaluados por indicadores que correspondan al contexto regional y local (Chávez, 2012).

La ciudad de Carhuaz ubicada en la margen derecha del río Santa, se ha localizado sobre un cono ancho de deyección generado por derrumbes de rocas y avalanchas de nieve de la cordillera Blanca a la cabecera del río Chucchun. Durante el terremoto de 1970 sufrió una destrucción considerada en el 100\% de su territorio, no sólo como consecuencia del movimiento sísmico, sino también por las inundaciones que produjo el desborde del río Santa. Se estima que la mitad de sus habitantes murieron o resultaron heridos, ya que sobre una población calculada en 9,457 habitantes, hubieron 311 muertos, 235 desaparecidos y 4,307 heridos (INDECI, 2004)

En el Mapa de calificación de provincias según niveles de peligros sísmicos, la provincia de Carhuaz, se encuentra en la zona de peligro muy alto (Ver figura 2).

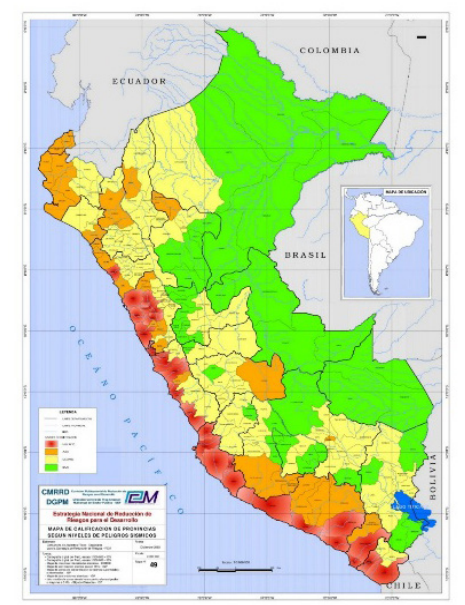

Figura 2. Mapa de calificación de provincias, según niveles de Peligro - CMRRD, 2003 MVCS 
Más del 60\% de las viviendas están construidas con tierra, la mayor parte de ellas con adobe por autoconstrucción, sin considerar los requisitos establecidos en las Normas vigentes en el Perú (Moromi, 2012).

El terremoto del 31 de mayo de 1970 ocurrido en Ancash, Perú, con una magnitud de 8,2 paso a la historia como el evento más mortífero ocurrido en las Américas, durante el siglo XX, más de 67 mil víctimas fue el resultado de esta tragedia.

Está comprobado que el silencio sísmico existente en zonas expuestas a fenómenos naturales intensos de origen geológico, si bien no permite predecir el lugar exacto donde ocurrirá un terremoto y mucho menos la fecha, si posibilita asegurar en que macroregión sísmica, tarde o temprano, ocurrirá una poderosa descarga de energía acumulada, que producirá un sismo de gran magnitud (Kuroiwa, 2010).

Debido al evento ocurrido el 11 de abril del 2010, por el desembalse de la laguna 513, ubicada en la cuenca alta de la Subcuenca Chucchun, se realizó el mapeo y modelamiento de amenazas y otros estudios. En los estudios se consideran que un posible desastre no solo está condicionado por la ocurrencia de un aluvión, éste se asocia a las condiciones de vulnerabilidad existentes principalmente relacionada a la vulnerabilidad de los factores físicos, sociales, económicos y ambientales. Los factores físicos se relacionan con el diseño, tipo, calidad de construcción y ubicación entre otros de las viviendas (Valderrama y Vilca, 2012).

En el marco de la Ley N²9664 del Sistema Nacional de Gestión del Riesgo de Desastres y su Reglamento (D.S. N048-2011-PCM) se define la vulnerabilidad como la susceptibilidad de la población, la estructura física o las actividades socioeconómicas, de sufrir daños por acción de un peligro o amenaza. (CENEPRED, 2014)

En este contexto, considerando que el peligro sismo es constante y que de ocurrir puede causar graves desastres, el estudio de la vulnerabilidad estructural de las viviendas expuestas en el área de influencia ante riesgo sísmico en la subcuenca Chucchun contribuye con la gestión del riesgo de desastres y permite proteger la vida y bienes materiales de las personas, aspectos del desarrollo sostenible.

\section{MATERIALES Y MÉTODOS}

Se aplicó el diseño no experimental, el trabajo se realizó en tres etapas; se realizaron visitas a campo y revisión de la información de fuentes secundarias de instituciones como el IGP, INGEMMET, CENEPRED, etc, para la identificación de las condiciones del territorio; para la identificación de las características de los indicadores que influyen en la vulnerabilidad estructural de las viviendas se aplicaron 343 encuestas, asimismo para la determinación de los niveles de vulnerabilidad estructural de las viviendas ante el riesgo sísmico, se empleó el método multicriterio, Proceso de Análisis 
Jerárquico (PAJ) y con la escala de Saaty, se ponderaron los criterios y descriptores seleccionados en las matrices de comparación y normalización.

\section{RESULTADOS}

Los factores que influyen en la vulnerabilidad de las viviendas, están relacionadas con las características del suelo, localización y pendiente donde se ubican, los resultados se presentan en las siguientes figuras.

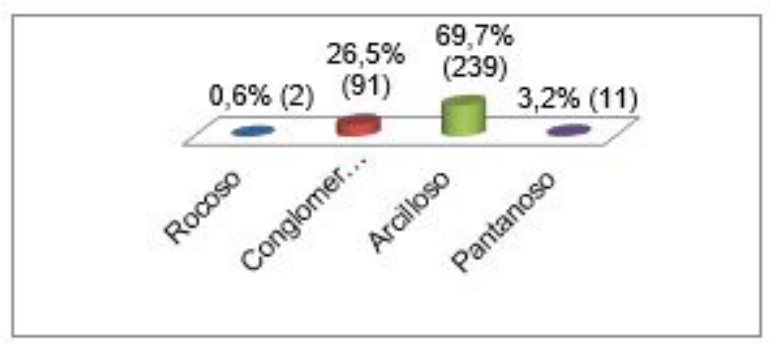

Figura 3. Características del suelo

Según la figura 3, las características del suelo donde se ubican las viviendas, se determinó que 239 (69,7\%) están sobre suelo arcilloso, 91 viviendas (26,5\%), en suelo conglomerado, $11(3,2 \%)$ sobre suelo pantanoso y $2(0,6 \%)$ sobre un suelo rocoso.

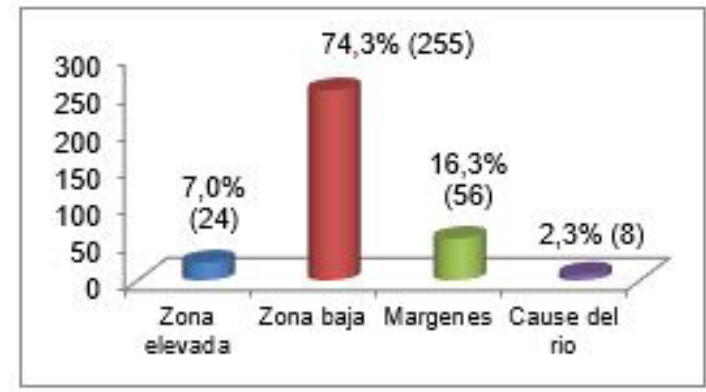

Figura 4. Localización de ls viviendas

En la figura 4, se muestra que $255(74,3 \%)$ se encuentran en zona baja, $56(6,3 \%)$ en las márgenes de ríos, 24 (7,0\%) en zona elevada y 8 (2,3\%) están localizadas en cauce del río 


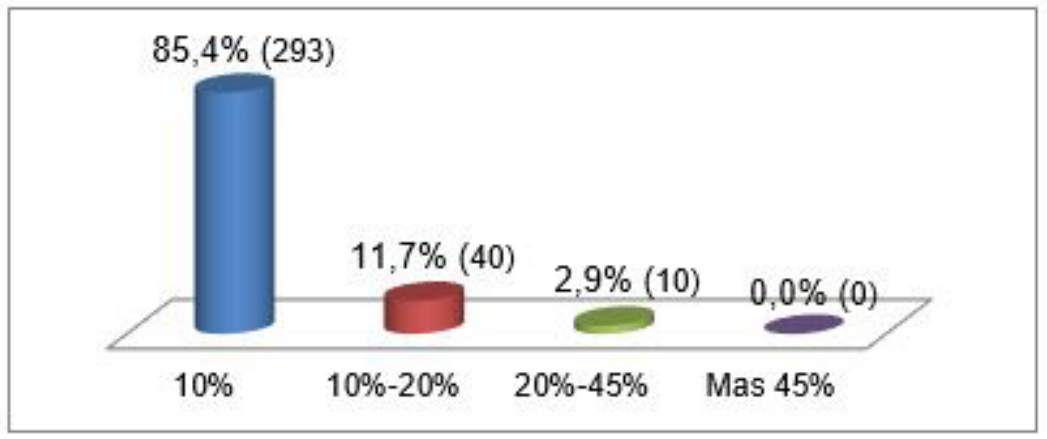

Figura 5. Pendiente donde se ubican las viviendas

Con respecto a la pendiente, en la figura 5 se muestra que, 293 viviendas $(85,4 \%)$ están construidas sobre una pendiente de hasta el 10\%, 40 (11,7\%) están construidas sobre una pendiente moderada de $10 \%$ al 20\%, $10(2,9 \%)$ en pendiente pronunciada de $20 \%$ al $45 \%$.

Las características de las variables e indicadores que influyen en la vulnerabilidad estructural de las viviendas de la Subcuenca Chucchun ante riesgo sísmico, se determinaron aplicando 343 encuestas, en las siguientes figuras se presentan los resultados:

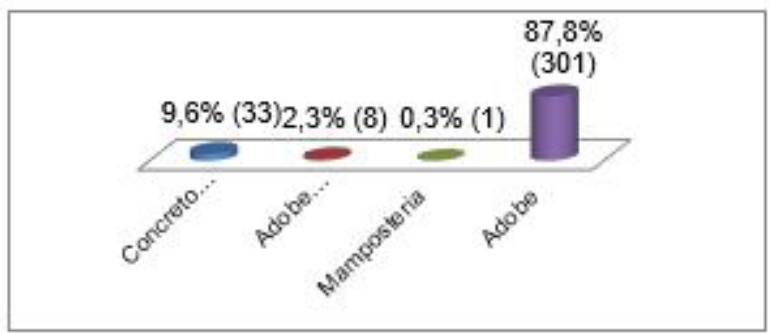

Figura 6. Tipo de material de la viviendas

301 viviendas $(87,8 \%)$ son de material adobe, $33(9,6 \%)$ de concreto armado, $8(2,3 \%)$ de adobe reforzado y $1 \quad(0,3 \%)$ es de mampostería

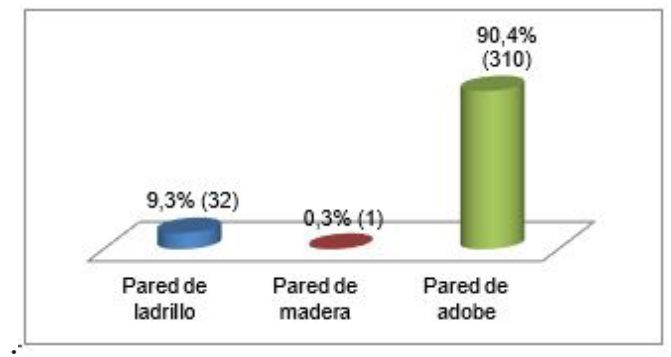

Figura 7. Tipo de material de las paredes 
En la figura 7, en las paredes de 310 viviendas (90,4\%), el material predominante es el adobe, $32(9,3 \%)$ de ladrillo y $1(0,3 \%)$ tiene pared de madera.

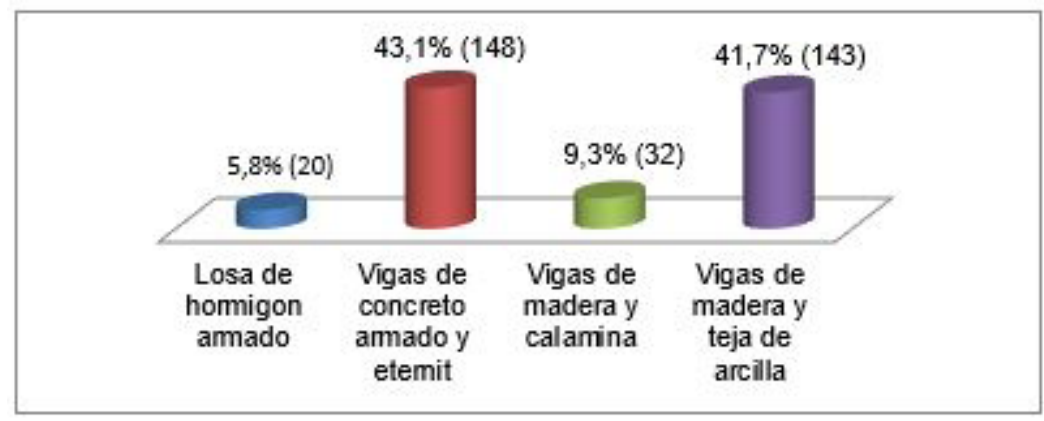

Figura 8. Tipo de cubierta de las viviendas

En la figura 8, se muestran los resultados del tipo de material utilizado como sistema de cubierta en las viviendas, 148 (43,1\%) tienen vigas de concreto armado y eternit, $143(41,7 \%)$ tienen vigas de madera y teja de arcilla, $32(9,3 \%)$ tienen vigas de madera y calamina y $20(5,8 \%)$ tienen losa de concreto armado como cubierta.

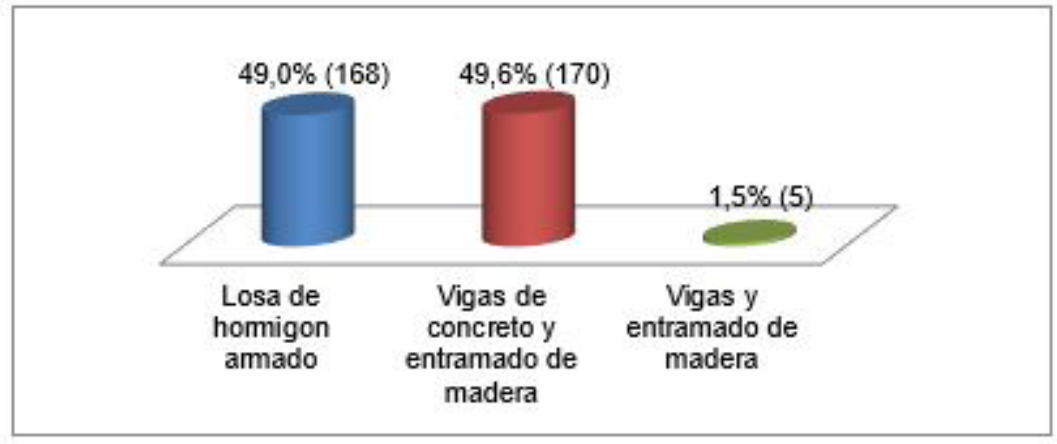

Figura 9. Sistema de entrepisos de las viviendas

La figura 9, muestra los resultados del tipo de material utilizado para el sistema de entrepisos de las viviendas, de las cuales 170 (49,6\%) tienen vigas de concreto y entramado de madera, $168(49,0 \%)$ tienen losa de concreto armado y solamente $5(1,5 \%)$ tienen vigas y entramado de madera. 


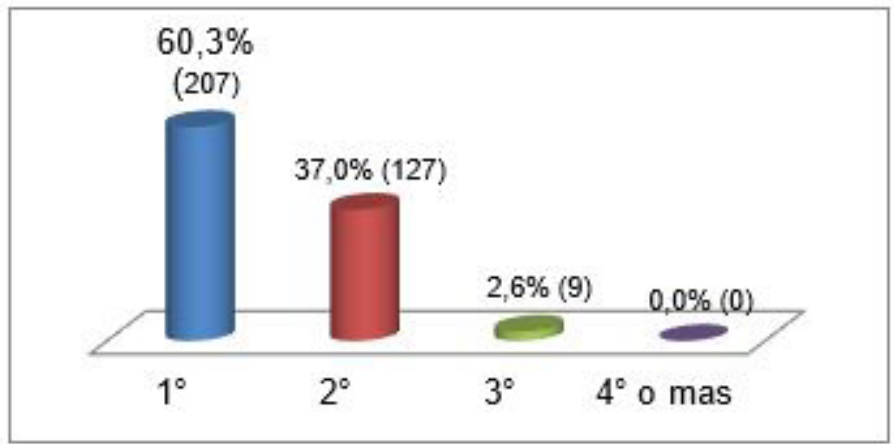

Figura 10. Número de pisos de las viviendas

La figura 10, muestra los resultados del numero de pisos de las viviendas, 207 (60,3\%) son de un piso, $127(37,0 \%)$ tienen 2 pisos y $9(2,6 \%)$ tienen 3 pisos.

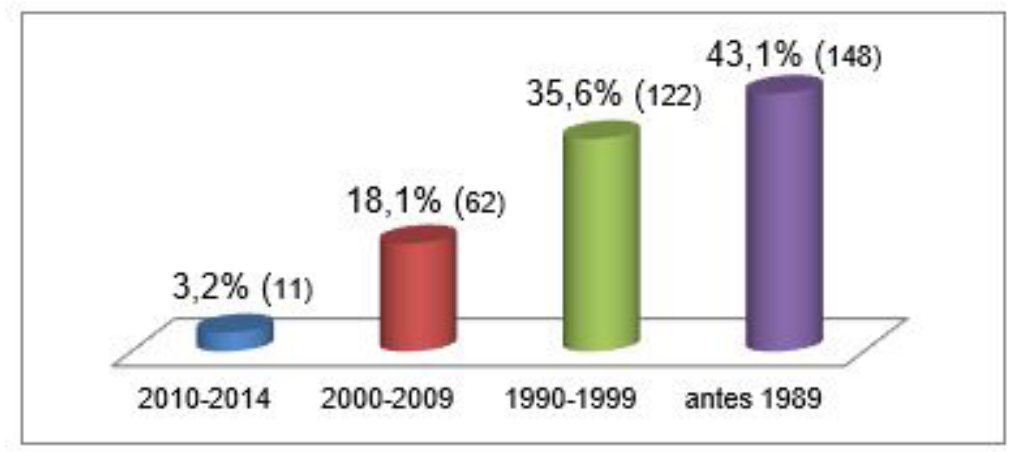

Figura 11. Año de construcción

Según la figura 11, 148 viviendas (43,1\% ) han sido construidas antes del año 1989, 122 (35,6\% ) han sido construidas entre los años 1990 a 1999, 62 (18,1\% ) han sido construidas entre los años 2000 a 2009 y 11 viviendas (3,2\%) han sido construidas entre los años 2010 a 2014.

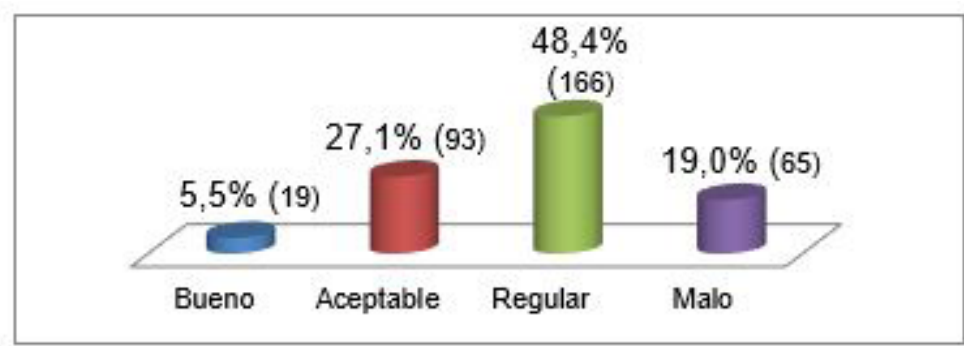

Figura 12. Estado de conservación 
En la figura 12, 166 viviendas (48,4\%) tienen un estado de conservación regular, 93 $(27,1 \%)$ tienen un estado de conservación aceptable, 65 (19,0\%) tienen un estado de conservación mala y 19 viviendas $(5,5 \%)$ tienen un estado de conservación bueno.

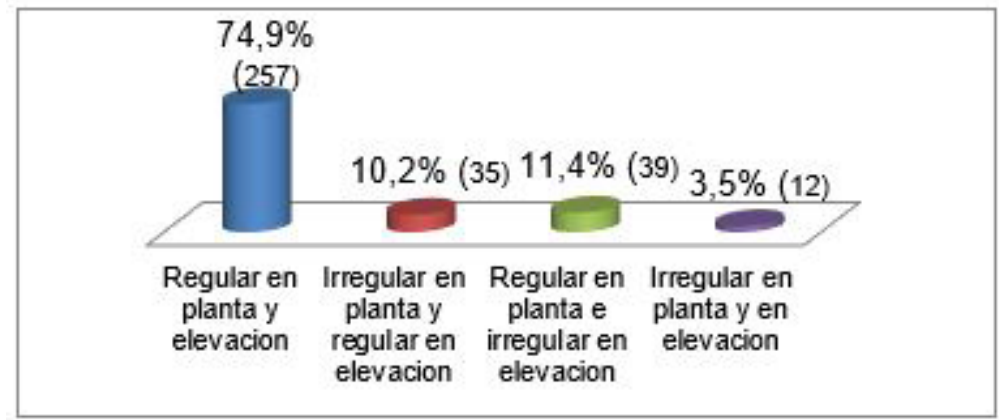

Figura 13. Forma de construcción

En la figura 13, 257 viviendas (74,9\%), presentan forma de construcción regular en planta y elevación, 39 (11,4\%), son regular en planta e irregular en elevación, 35 (10,2\%) son irregular en planta y regular en elevación y 12 viviendas (3,5\%), presentan forma de construcción irregular en planta y elevación.

Los resultados del nivel de la vulnerabilidad estructural ante riesgo sísmico de las viviendas se presentan en las siguientes tablas:

Tabla 1. Matríz de comparación de pares de la vulnerabilidad estructural de las viviendas

\begin{tabular}{|c|c|c|c|c|c|c|c|c|}
\hline $\begin{array}{c}\text { Variables de vulne- } \\
\text { rabilidad en edifica- } \\
\text { ciones }\end{array}$ & $\begin{array}{c}\text { Localiza- } \\
\text { ción de la } \\
\text { vivienda }\end{array}$ & $\begin{array}{c}\text { Tipo de } \\
\text { material en } \\
\text { paredes }\end{array}$ & $\begin{array}{c}\text { Material } \\
\text { techo }\end{array}$ & $\begin{array}{c}\text { Material } \\
\text { piso }\end{array}$ & $\begin{array}{c}\text { Número } \\
\text { de pisos }\end{array}$ & $\begin{array}{c}\text { Año } \\
\text { construc- } \\
\text { ción }\end{array}$ & $\begin{array}{c}\text { Estado de } \\
\text { conserva- } \\
\text { ción }\end{array}$ & $\begin{array}{c}\text { Topografía } \\
\text { del sitio }\end{array}$ \\
\hline $\begin{array}{c}\text { Localización de la } \\
\text { vivienda }\end{array}$ & 1,00 & 0,33 & 0,50 & 2,00 & 0,50 & 3,00 & 0,50 & 2,00 \\
\hline $\begin{array}{c}\text { Tipo de material en } \\
\text { paredes }\end{array}$ & 3,00 & 1,00 & 3,00 & 9,00 & 2,00 & 5,00 & 2,00 & 3,00 \\
\hline Material techo & 2,00 & 0,33 & 1,00 & 5,00 & 0,50 & 2,00 & 3,00 & 4,00 \\
\hline Material piso & 0,50 & 0,11 & 0,20 & 1,00 & 0,20 & 0,50 & 0,25 & 0,50 \\
\hline Número de pisos & 2,00 & 0,50 & 2,00 & 5,00 & 1,00 & 2,00 & 3,00 & 4,00 \\
\hline Año construcción & 0,33 & 0,20 & 0,50 & 2,00 & 0,50 & 1,00 & 0,50 & 2,00 \\
\hline Estado de conser- & 2,00 & 0,50 & 0,33 & 4,00 & 0,33 & 2,00 & 1,00 & 2,00 \\
\hline vación & 0,50 & 0,33 & 0,25 & 2,00 & 0,25 & 0,50 & 0,50 & 1,00 \\
\hline Topografía del sitio & 11,33 & 3,31 & 7,78 & 30,00 & 5,28 & 16,00 & 10,75 & 18,50 \\
\hline SUMA & 0,09 & 0,30 & 0,13 & 0,03 & 0,19 & 0,06 & 0,09 & 0,05 \\
\hline 1/SUMA & & & & & & \\
\hline
\end{tabular}


Tabla 2. Matríz de normalización de pares de la vulnerabilidad estructural de las viviendas

\begin{tabular}{|c|c|c|c|c|c|c|c|c|c|}
\hline $\begin{array}{c}\text { Variables de } \\
\text { vulnerabilidad } \\
\text { en edificacio- } \\
\text { nes }\end{array}$ & $\begin{array}{l}\text { Localiza- } \\
\text { ción de la } \\
\text { vivienda }\end{array}$ & $\begin{array}{l}\text { Tipo de } \\
\text { material } \\
\text { en pare- } \\
\text { des }\end{array}$ & $\begin{array}{l}\text { Material } \\
\text { techo }\end{array}$ & $\begin{array}{c}\text { Material } \\
\text { piso }\end{array}$ & $\begin{array}{l}\text { Número } \\
\text { de pisos }\end{array}$ & $\begin{array}{c}\text { Año } \\
\text { construc- } \\
\text { ción }\end{array}$ & $\begin{array}{c}\text { Estado de } \\
\text { conserva- } \\
\text { ción }\end{array}$ & $\begin{array}{c}\text { Topografía } \\
\text { del sitio }\end{array}$ & $\begin{array}{l}\text { Vector } \\
\text { Priori- } \\
\text { zacion }\end{array}$ \\
\hline $\begin{array}{l}\text { Localización } \\
\text { de la vivienda }\end{array}$ & 0,088 & 0,101 & 0,064 & 0,067 & 0,095 & 0,188 & 0,047 & 0,108 & 0,095 \\
\hline $\begin{array}{l}\text { Tipo de mate- } \\
\text { rial en paredes }\end{array}$ & 0,265 & 0,302 & 0,385 & 0,300 & 0,379 & 0,313 & 0,186 & 0,162 & 0,286 \\
\hline Material techo & 0,176 & 0,101 & 0,128 & 0,167 & 0,095 & 0,125 & 0,279 & 0,216 & 0,161 \\
\hline Material piso & 0,044 & 0,034 & 0,026 & 0,033 & 0,038 & 0,031 & 0,023 & 0,027 & 0,032 \\
\hline $\begin{array}{c}\text { Número de } \\
\text { pisos }\end{array}$ & 0,176 & 0,151 & 0,257 & 0,167 & 0,189 & 0,125 & 0,279 & 0,216 & 0,195 \\
\hline $\begin{array}{c}\text { Año construc- } \\
\text { ción }\end{array}$ & 0,029 & 0,060 & 0,064 & 0,067 & 0,095 & 0,063 & 0,047 & 0,108 & 0,067 \\
\hline $\begin{array}{c}\text { Estado de } \\
\text { conservación }\end{array}$ & 0,176 & 0,151 & 0,043 & 0,133 & 0,063 & 0,125 & 0,093 & 0,108 & 0,112 \\
\hline $\begin{array}{c}\text { Topografía del } \\
\text { sitio }\end{array}$ & 0,044 & 0,101 & 0,032 & 0,067 & 0,047 & 0,031 & 0,047 & 0,054 & 0,053 \\
\hline
\end{tabular}

Tabla 3. Cálculo de la vulnerabilidad

\begin{tabular}{|c|c|c|c|c|c|c|c|c|c|c|c|c|c|c|c|c|}
\hline \multicolumn{17}{|c|}{ Vulnerabilidad } \\
\hline \multicolumn{2}{|c|}{$\begin{array}{l}\text { Localización } \\
\text { de la vivienda }\end{array}$} & \multicolumn{2}{|c|}{$\begin{array}{c}\text { Tipo de } \\
\text { material en } \\
\text { paredes }\end{array}$} & \multicolumn{2}{|c|}{ Material techo } & \multicolumn{2}{|c|}{ Material piso } & \multicolumn{2}{|c|}{$\begin{array}{c}\text { Número de } \\
\text { pisos }\end{array}$} & \multicolumn{2}{|c|}{$\begin{array}{l}\text { Año cons- } \\
\text { trucción }\end{array}$} & \multicolumn{2}{|c|}{$\begin{array}{l}\text { Estado de } \\
\text { conservación }\end{array}$} & \multicolumn{2}{|c|}{$\begin{array}{c}\text { Topografía } \\
\text { del sitio }\end{array}$} & \multirow{2}{*}{ Valor } \\
\hline 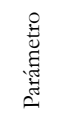 & 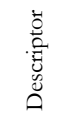 & 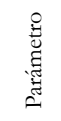 & 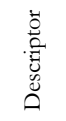 & 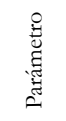 & 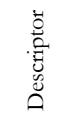 & 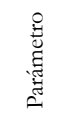 & 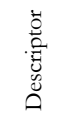 & 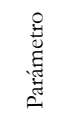 & 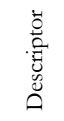 & 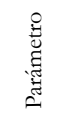 & 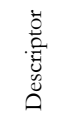 & 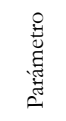 & 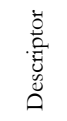 & 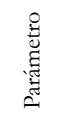 & 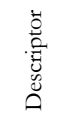 & \\
\hline 0,095 & 0,503 & 0,286 & 0,430 & 0,161 & 0,105 & 0,032 & 0,408 & $0 ., 195$ & 0,068 & 0,067 & 0,088 & 0,112 & 0,149 & 0,053 & 0,256 & 0,250 \\
\hline
\end{tabular}

Tabla 4. Niveles de vulnerabilidad

\begin{tabular}{|c|c|}
\hline \multicolumn{2}{|c|}{ NIVELES VULNERABILIDAD } \\
\hline $0,271 \leq \mathrm{V} \leq 0,457$ & MUY ALTA \\
\hline $0,155 \leq \mathrm{V} \leq 0,271$ & ALTA \\
\hline $0,078 \leq \mathrm{V} \leq 0,155$ & MEDIA \\
\hline $0,039<\mathrm{V} \leq 0,078$ & BAJA \\
\hline
\end{tabular}




\section{DISCUSIÓN}

De los resultados obtenidos se evidencia que las viviendas localizadas en suelos arcillosos y en zonas de pendiente alta, son mas vulnerables y se encuentran expuestas ante un riesgo símicos, esta información concuerda con la información del IGP y se muestra en el mapa sísmico del Perú y con la información del Mapa de peligros presentado por el Ministerio de Vivienda, Construcción y Saneamiento.

Los resultados obtenidos de la aplicación de encuestas muestran que las características de las variables e indicadores de las viviendas como el tipo de material de las viviendas, de paredes, de cobertura, de entepisos, de número de pisos, del año de construcción y estado de conservación, influyen en la vulnerabilidad estructural ante riesgo sísmico, los cuales se correlacionan con los presentados por Laucala (2013), quien recolectó datos de ubicación, proceso constructivo, estructuración y calidad de la construcción y luego en gabinete procesó la información elaborando un análisis sísmico simplificado por medio de la densidad de muros, determinando la vulnerabilidad, peligro y riesgo sísmico de las viviendas encuestadas.

Apply technology council (2005), realizó una evaluación rápida, con estimación de daños producidos en las edificaciones luego de un sismo y no estimó la vulnerabilidad de edificaciones existentes, estos resultados contrastan con los obtenidos en la presente investigación que tuvo por objetivo determinar la vulnerabilidad estructural de las viendas ante la probabilidad de ocurrencia del peligro sísmico.

Con respecto a la determinación del nivel de vulnerabilidad estructural de viviendas ante riesgo sísmico con el método aplicado en la presente investigación, no se encontraron investigaciones similares, situación que se justifica porque el Centro Nacional de Estimación, Prevención y Reducción del Riesgo de Desastres, ente asesor, técnico normativo de la gestión del riesgo de desastres, recientemente ha editado el manual de evaluación del riesgo de desastres ante fenómenos naturales, para promover las investigaciones aplicando el mencionado método, a través del cual se determinan condiciones de peligrosidad, vulnerabilidad y riesgo y proponer medidas de prevención y reducción del riesgo de desastres para salvaguardar la vida de la población, protección de su infraestrucrua física y su entorno ambiental.

\section{CONCLUSIONES}

Las viviendas se ubican mayormente en suelos arcillosos, en las partes bajas y en pendiente ligera, condiciones que asociadas a la ubicación geográfica de la subcuenta, en una zona altamente sísmica, y al silencio sísmico, condicionan el riesgo de colapso de las viviendas ante la ocurrencia de un sismo.

Las características de las variables e indicadores que influyen en la vulnerabilidad estructural de las viviendas de la Subcuenca Chucchun ante riesgo sísmico, se encuentran asociadas a la antigüedad, tipo de material, número de pisos, cobertura, sistema de entrepisos que de acuerdo a los resultados presentan vulnerabilidad ante la ocurrencia de un sismo. 
Las viviendas evaluadas presentan niveles índice de vulnerabilidad alta y considerando que el riesgo es el resultado de relacionar el peligro con la vulnerabilidad de los elementos expuestos, ante la ocurrencia de un sismo de mayor magnitud el riesgo es alto y en consecuencia se generaría un desastre con grandes pérdidas humanas y materiales en las localidades de la subcuenca Chucchun.

\section{REFERENCIAS BIBLIOGRÁFICAS}

Apply Technology council. 2005. Método ATC-20 rapid evaluation safety assessment form. $<$ https://www.atcouncil.org/pdfs/rapid.pdf $>$ [Consulta:12-04.2017].

Chávez, J. 2012. “Consultoría para la definición operativa y diseño del cuestionario para la medición de indicadores de resultado del Programa Presupuestario Reducción de la Vulnerabilidad y Atención de Emergencias por Desastres en el componente de Resiliencia". Perú.

CENEPRED. 2014. Manual para la evaluación de riesgos originados por fenómenos naturales, Cenepred, Lima-Perú

Instituto Geofísico del Perú. 2011. "El Geofísico".< http://repositorio.igp.gob. pe/handle/IGP/874> [Consulta:12-03.2017].

INDECI. 2004. "Mapa de peligros - Plan de usos del suelo y medidas de mitigación ante desastres". Carhuaz, Ancash.

Kuroiwa, Julio. 2010. Alto a los desastres. Viviendas seguras y saludables para los peruanos con menores recursos. Primera edición. Lima:Unmbral Ediciones SAC.

Laucala, Johan. 2013. Análisis de la vulnerabilidad sísmica de las viviendas informales en la ciudad de Trujillo, PUCP. Lima, Perú

Moromi, Isabel. 2012. Gestión del Riesgo: Metodología para la evaluación de la vulnerabilidad sísmica de edificaciones de adobe a nivel local. Tesis de maestría, UNI, Lima. Perú.

Valderrama, Patricio; Vilca, Oscar, 2012. Dinámica e implicancia del aluvión de la laguna 513, Cordillera Blanca, Ancash, Perú, Revista de la Asociación Geológica Argentina, Buenos Aires -Argentina.

Salzmann, et al. 2016. "Climate change adaptation Strategies - An Upstream-downstream Perspective”. 2016. PUCP. Lima Perú.

Recepción: 20/09/2018

Aceptación: 10/11/2018

\section{Correspondencia}

Rosa Deifilia Rodríguez Anaya

rosanaya2012@gmail.com 